\title{
Prognostic factors in endometrial cancer
}

\section{Bajracharya S R, Juan F Y}

Department of Obstetrics and Gynecology, Tribhuwan University Teaching Hospital, Kathmandu

Correspondence address: Dr. Sunita Rajbhandari Bajracharya, Department of Obste-trics and Gynecology, TU Teaching Hospital, Kathmandu, Nepal

Email: dsrajbhandari@hotmail.com

\begin{abstract}
Introduction: Endometrial cancer (EC) is the most common malignancy of the female genital tract. It is now the fourth most common gynecological cancer among women and the sixth worldwide cancer in Western countries. It is the most curable of the 10 most common cancers in women and the most frequent and curable of the gynecologic cancers. The incidence is increasing when life expectancy rise. This study was carried out to evaluate the prognostic factors of Endometrial Cancer.

Methods: Clinico-pathological characteristics and follow-up cases of endometrial cancer were analysed retrospectively between January 2000 to December 2007 with its prognostic factors influence were statistically analysed.

Results: During the study period total 180 patients were taken. Univariate model revealed that the menopausal status, the FIGO stage, grade, histological type, myometrial invasion, ER, PR, peritoneal cytology, lymph node metastases, adjuvant therapy, and the method of operation were related with the prognosis significantly. The multivariate analysis Cox proportion hazards regression model showed that the $\mathrm{ER}(\mathrm{P}=0.004), \mathrm{PR}(\mathrm{P}=0.000)$, myometrial invasion $(\mathrm{P}=0.006)$ and lymph node metastases $(\mathrm{P}=0.049)$ were related with the prognosis significantly.

Conclusion: The clinico- pathological character of endometrial cancer is responsible for patient's better survival. If the patients had early detection and treated in proper modalities, this might improve the good prognosis.
\end{abstract}

Keywords: endometrial cancer, prognosis, prognostic factors

\section{Introduction}

Endometrial Cancer (EC) is one of the three malignant tumours of the female reproductive system. The incidence increases steadily with the age and peaks between the age 70-74 years. Fortunately, the prognosis for survival is high: the 5 year survival in Sweden is over $80 \% .^{1,2}$ Ninety Percentage of all cancers of the uterus arise from the glands of the endometrium so called as endometrial adenocarcinoma. ${ }^{3-6}$ Type I EC is hormone dependent, about $80 \%$ is characte-rized by well to moderately differentiated tumors that present at diagnosis and therefore the overall prognosis is optimistic. Excessive estrogen is associated with most of the risk factors that is linked to endometrial carcinoma. Patients with atypical endometrial hyperplasia have a $23 \%$ of risk of endometrial carcinoma over ten years. The Type II is non hormone dependent, about $23 \%$ characterized by more aggressive tumours usually not associated with unopposed estrogens or hyperplasia and shows a high mortality rate with poorer prognosis. During the past two decades, there are improvements in treatment and its follow-up of endometrial car-cinoma patients, the importance of various prognostic factors has been extensively studied and published the prognostic parameters of endometrial cancer. These factors are important in tailoring proper adjuvant therapy consisting of modalities such as radiation, chemotherapy and hormonal therapy. Over the past few decades, several studies have demonstrated the prognostic importance of different parameters including lymph node status, histological type of carcinoma, histological grade and stage of disease, depth of myometrial invasion, lympho-vascular space involvement and cervical involvement.

This study aims to review the Clinical pathological materials as prognostic factors of endo-metrial cancer. 
This will enables the high risk patients to obtain suitable moderate treatment and avoids recurrence and metastasis and improve diseases free survival rate and quality of life of women.

\section{Methods}

This was retrospective study of patients admitted from Jan 2000 to Dec 2007 in gynecologi-cal department of Guangxi Medical University with the diagnosis of Endometrial Carcinoma who underwent primary surgical procedure were taken as cases. Hospital charts records of these cases were reviewed and updated by use of modified 2000 FIGO criteria for Endome-trial Carcinoma. These patients were followed up till 2008 Dec 31.

The follow up was done by telephone inquiry and letter correspondence. If there was no fol-low up for up to four times, and no reply by telephone calls, then the information of the local police station or local administration for death records were checked, If there was no infor-mation, the patients was taken as lost to follow up. SPSS 13.0 software was used for analysis of prognostic factors of endometrial cancer. Univariate analysis was estimated by Log-rank test, and the multivariate analysis was assessed by Cox proportion hazards regression model. $\mathrm{P}$ value $<0.05$ was taken as significant.

\section{Result}

There were 190 patients admitted in the gynecological department of Guangxi Medical Uni-versity with the diagnosis of Endometrial Carcinoma. During the study period 180 patients underwent primary surgical procedure. Ten patients were excluded as four refused for prima-ry surgical procedure and six patients did not undergo the surgical procedure, as four were of late stage, one of which had heart disease and the other had surgically not fit due to old age.

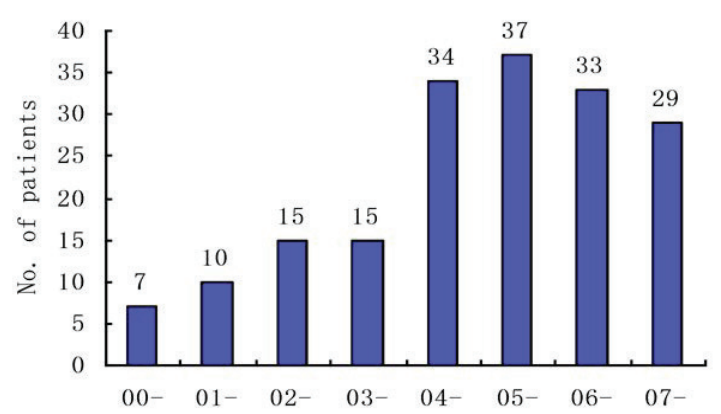

Figure 1 Distribution of total no of endometrial cancer patient admitted in each year

Figure 1 shows distribution of Endometrial Carcinoma in each year. There were more patients from 2004 onwards. The median age of the patients was 53 yrs (30-71years), and average age of menopause was 50.6 yrs (41-59yrs).

Table 1: Univariate prognostic factors analysis of endometrial cancer patients according to demographic profile.

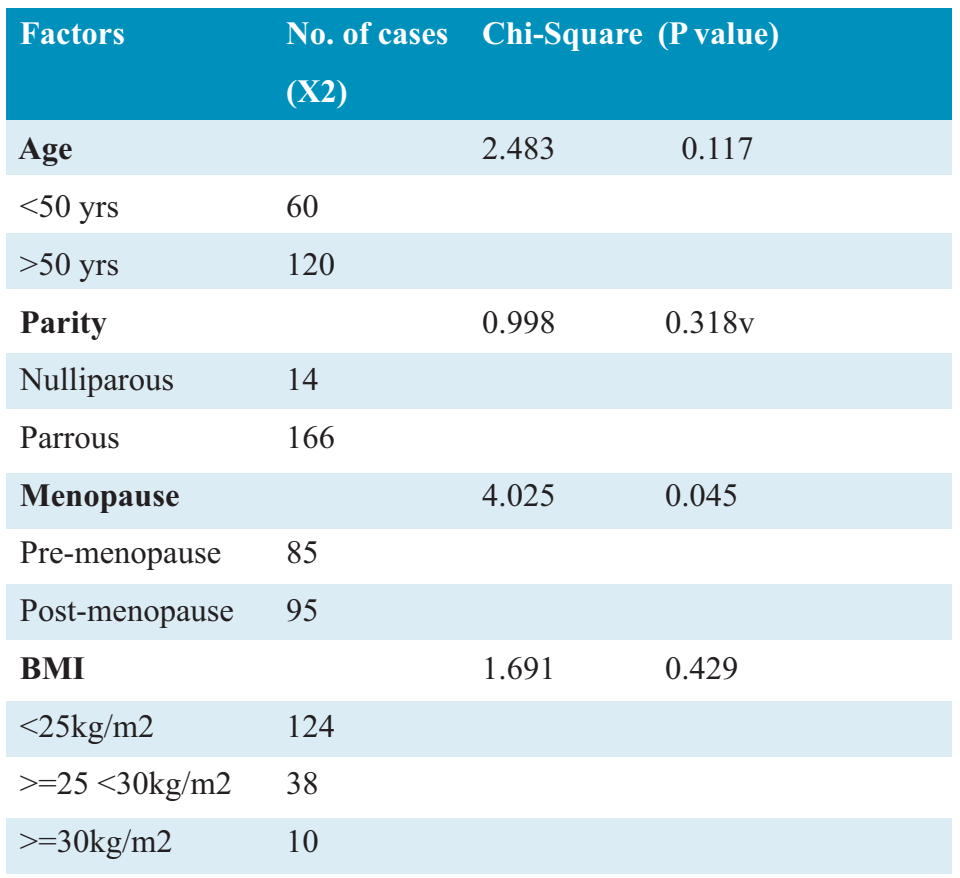

Table 1 shows Univariate prognostic factors analysis of endometrial cancer patients according to demographic profile where menopausal status was significant $(\mathrm{P}<0.05)$ for the prognosis of the endometrial cancer, whereas parity, age and BMI were not significant.

Table 2: Univariate prognostic factors analysis of endometrial cancer patients according to Associate medical disorder.

\begin{tabular}{llll}
$\begin{array}{l}\text { Factors } \\
\text { No. of cases }\end{array}$ & $\begin{array}{c}\text { Chi-Square } \\
\text { (X2) }\end{array}$ & Sig. (P value) \\
\hline Associate & & & \\
diseases & & 2.431 & 0.488 \\
\hline No diseases & 143 & & \\
\hline Dibetes & & \\
\hline Mellitus(DM) & 11 & \\
\hline Hypertension & \\
\hline (HTN) & 20 \\
\hline DM+HTN & 6
\end{tabular}


Table 2 shows Univariate prognostic factors analysis of endometrial cancer patients according to associate medical disorder which was not significant $\mathrm{P}>0.05$ for the prognosis of the endometrial cancer.

Table 3: Univariate prognostic factors analysis of endometrial cancer patients according to Surgical stage.

\begin{tabular}{|c|c|c|c|}
\hline Factors & No. of cases & Chi-Square (X2) & Sig. (P value) \\
\hline Surgical Stage & & 14.516 & 0.000 \\
\hline Stage I & 112 & & \\
\hline Stage II & 18 & & \\
\hline Stage III & 49 & & \\
\hline Stage IV & 1 & & \\
\hline
\end{tabular}

Table 3 shows Univariate prognostic factors analysis of endometrial cancer patients according to surgical stage which was significant $(\mathrm{P}<0.05)$ for the prognosis of the endometrial cancer.

Table 4: Univariate prognostic factors analysis of endometrial cancer patients according to Treatment.

\begin{tabular}{|c|c|c|c|}
\hline Factors & No. of cases & Chi-Square (X2) & Sig. (P value) \\
\hline Surgical Procedure & & 7.906 & 0.045 \\
\hline $\mathrm{TAH}+/-\mathrm{BSO}$ & 74 & & \\
\hline \multicolumn{4}{|l|}{ TAH+/-BSO \& Pelvic } \\
\hline Lymphadenectomy & 33 & & \\
\hline \multicolumn{4}{|l|}{ RH+/-BSO \& pelvic } \\
\hline Lymphadenectomy & 50 & & \\
\hline \multicolumn{4}{|l|}{ Semi RH+/- BSO \& } \\
\hline Pelvic Lymphadenectomy & 23 & & \\
\hline Adjuvant Therapy & & 1.028 & 0.905 \\
\hline No therapy & 74 & & \\
\hline Radiotherapy(RT) & 14 & & \\
\hline Chemotherapy(CT) & 62 & & \\
\hline Both $(\mathrm{RT}+\mathrm{CT})$ & & & \\
\hline Hormonal Therapy 12 & & & \\
\hline
\end{tabular}

Table 4 shows Univariate prognostic factors analysis of endometrial cancer patients according to treatment procedure where surgical procedure was significant $(\mathrm{P}<0.05)$ for the prognosis of the endometrial cancer. 
Table 5: Univariate prognostic factors analysis of endometrial cancer patients according to Pathological findings,

\begin{tabular}{|c|c|c|c|}
\hline Factors & No. of cases & Chi-Square (X2) & Sig. (P value) \\
\hline Histopathological Grade & & 6.269 & 0.044 \\
\hline G1 & 84 & & \\
\hline G2 & 72 & & \\
\hline G3 & 24 & & \\
\hline Myometrial Invasion & & 11.874 & 0.000 \\
\hline$<1 / 2$ & 139 & & \\
\hline$>1 / 2$ & 41 & & \\
\hline \multicolumn{4}{|l|}{ Steroid Hormone Receptor } \\
\hline ER & & 4.901 & 0.002 \\
\hline Positive & 119 & & \\
\hline Negative & 61 & & \\
\hline PR & & 20.046 & 0.000 \\
\hline Positive & 129 & & \\
\hline Negative & 51 & & \\
\hline Histological Type & & 9.662 & 0.022 \\
\hline Endoometrial adenocarcinoma(EA) & 135 & & \\
\hline EA with squamous adenocarcinoma & 19 & & \\
\hline Papillary serous carcinam & 25 & & \\
\hline Squamous carcinaoma & 1 & & \\
\hline Peritoneal cytology & & 5.554 & 0.000 \\
\hline Positive & 29 & & \\
\hline Negative/Not sample & 151 & & \\
\hline Lymphnode metastases & & 6.852 & 0.033 \\
\hline Metastases & 7 & & \\
\hline No metastases & 102 & & \\
\hline Not sampled & 71 & & \\
\hline
\end{tabular}

Table 5 shows Univariate prognostic factors analysis of endometrial cancer patients according to treatment procedure where histopathological grade, myometrial invasion, ER, PR , histological type, peritoneal cytology and lymphnode metastases were significant $(\mathrm{P}<0.05)$ for the prognosis of the endometrial cancer. 
Table 6: Cox proportional hazard regression model for multivariate prognostic factors of endometrial cancer

\begin{tabular}{|lccccccccc|}
\hline & B & SE & Wald & df & Sig. & OR & \multicolumn{2}{c|}{ OR 95.0\% CI } \\
\hline ER & & & & & & & & Lower Upper \\
\hline PR & 1.130 & .387 & 8.518 & 1 & .004 & 3.096 & 1.449 & 6.611 \\
\hline Myometrial Invasion & -1.610 & .312 & 26.649 & 1 & .000 & .200 & .108 & .368 \\
\hline Surgical Stage(FIGO) & .852 & .308 & 7.671 & 1 & .006 & 2.345 & 1.283 & 4.285 \\
\hline Lymphnode metastasis & .061 & .237 & 0.66 & 1 & .797 & 1.063 & 1.691 & 1.691 \\
\hline Menopause & -.582 & .309 & 3.547 & 1 & .049 & 1.853 & .953 & 3.605 \\
\hline Surgical Procedure & .268 & .341 & .619 & 1 & .432 & 1.307 & .671 & 2.549 \\
\hline Surgical grade & .026 & .191 & .019 & 1 & .891 & 1.027 & .706 & 1.493 \\
\hline Peritoneal cytology & .151 & .173 & .757 & 1 & .384 & 1.163 & .828 & 1.633 \\
\hline Pathological stage & .141 & .424 & .110 & 1 & .740 & 1.151 & .502 & 2.641 \\
\hline Adjuvant therapy & .220 & .221 & .992 & 1 & .319 & 1.246 & .808 & 1.920 \\
\hline
\end{tabular}

Table 6 shows multivariate prognostic factors of survival analysis in endometrial cancer. For Independent prognostic factors of endometrial cancer, which are comparatively significant with survival curve, we further analysis it by using Cox proportional hazard regression model which demonstrated ER, PR, myometrial invasion and lymphnode metastases has the remarkably significant for the prognosis of endometrial cancer.

\section{Discussion}

The prognosis of patients with endometrial carcinoma is well known to be related to histological grade and stage of tumor. According to the $\mathrm{GOG}^{7-10}$ the prognostic parameters for En-dometrial Carcinoma of the uterine factors include histological type, histological grade and depth of myometrial invasion, vascular invasion, presence of atypical endometrial hyperplasia, cervical involvement, DNA ploidy and S-shape fraction and hormone receptor status. The extra-uterine factors include positive peritoneal cytology, adnexal involvement, pelvic and para-aortic lymph node metastasis and peritoneal metastasis. Patients with extra-uterine disease along with cervical involvement and vascular invasion comprise a high risk group in which approximately $65 \%$ will experience recurrence ${ }^{10}$ and those patients with tumour con-fined to the uterus without evidence of vascular invasion have a decreased risk of recurrence. ${ }^{8-10}$

In this study, age less than 50 yrs and more than 50 yrs had no significant difference, though older age had a poor prognostic variable in endometrial cancer. Many authors have reported an inverse relationship between advancing age and outcome. ${ }^{11}$ More recently, Farley et al, ${ }^{12}$ demonstrated that endometrial cancer survival decreases significantly in older patients, with the 5 year survival falling below $80 \%$ after age 50. If a patient nulliparous and obese reaches menopause at age 52 or later, she appears to have a fivefold increase in the risk of developing endometrial cancer. Reduced 5-year survival of 57\% among nulliparous women in comparison with $81 \%$ for patients with one or more deliveries has been reported ${ }^{13}$ but there are no other studies to support this data. Though nulliparity is a risk factor for endometrial cancer, parity did not show effect in our study.

The degree of obesity generally has a proportional effect on a women's risk of endometrial cancer but BMI was not prognostic factor in this study. Epidemiological studies indicate that increased waist circumference and/or BMI are positively correlated with the development endometrial cancer (postmenopausal). ${ }^{14,15}$ Furthermore, a large prospective study by Calle and colleagues ${ }^{16}$ demonstrated that increasing BMI is associated with increased mortality from many types of cancer. The authors suggest that increased BMI ( $>$ $25 \mathrm{~kg} / \mathrm{m} 2$ ) contributes to 90,000 cancer-related deaths per year in the US.

Endometrial Carcinoma is often associated with medical disorders such as hyperten-sion, diabetes mellitus, obesity, PCOS, and infertility. ${ }^{17,18}$ Steiner et al, ${ }^{19}$ found $21.8 \%$ of 
patients with diabetes mellitus in endometrial carcinoma had a significantly deeper infiltration of the myometrium and were more likely to have lymph node metastasis. In this study, the effect of obesity, hypertension diabetes mellitus on endometrial carcinoma prognosis was not remarkable.

Both univariate and multivariate analysis showed a strong significant correlation between surgicopathological stages as prognostic factors for endometrial cancer. The FIGO staging system which is a surgical-pathological staging system..$^{20} \mathrm{Carol}^{21}$ reported 5 year disease free survival for stages I, II, III, IV, are $93.9 \%, 72.9 \%, 48.1 \%$ and $25.4 \%$. that conventional pathologic features do not lose their prognostic significance in pathologic stage I-II endometrial adenocarcinoma patients following Post-operative adjuvant radiation therapy, and 5 year disease free survival as $91.5 \%$ for stage IA / IB , $85.5 \%$ for stage IC, and $75.8 \%$ for stage IIA / IIB.

In this study, univariate analysis showed histological grade had significant prognostic effect but in multivariate analysis, difference was not significant. Large number of patients had low histological grade and few numbers with high grade. Histologicaliy, high grade with lymph node metastasis and myometrial invasion has influence on the survival rate of endo-metrial cancer. Five-year survival rates of $94 \%$ in patients with grade 1 tumors, $84 \%$ in those with grade 2 tumors and $72 \%$ in those with grade 3 tumors has been reported. ${ }^{22}$

Both univariate and multivariate analysis showed there was a significant correlation be-tween myometrial invasion as prognostic factors. In endometrial carcinomas the depth of myometrial invasion has been shown to be an independent predictor of outcome. ${ }^{7,10,23}$ Depth of myometrial invasion of tumor is correlated with a risk of lymph node metastases and an independent risk factor for risk of death and recurrence. 9,24 Patients with tumours that are less than $5 \mathrm{~mm}$ from the serosal surface are at much higher risk for recurrence and death than those with tumours greater than $5 \mathrm{~mm}$ from the serosal surface. ${ }^{5,6}$ In GOG Study 33, recurrence developed in only $1 \%$ of patients with no myometrial invasion. $7.7 \%$ had invasion of the inner one-third of the myometrium, $14.5 \%$ had invasion of the middle-third of the myo-metrium, and $15 \%$ had invasion of the outer-third of the myometrium. ${ }^{10}$

Univariate and multivariate analysis showed there was a significant correlation between steroid hormone receptor ER and PR as prognostic factors for endometrial cancer. Steroid hormones, especially estrogen, play an important role in the pathogenesis of endometrial can-cer. The presence and quantity of steroid receptors have been correlated with stage, histologi-cal grade and survival in several studies and several authors have reported that receptor status constitutes an independent prognostic factor. ${ }^{25,26}$ The United States National Cancer Insti-tute recommended that steroid receptors should be included whenever possible in the evalua-tion of stage I and II patients..$^{25}$

Only univariate analysis showed significance in histological types. This may be because there were large number of patients with low grade adnocarcinoma and minimally invasive with few numbers having serous cell and squamous cell carcinoma. The type I usually have a favorable outcome; stage I disease carries a 5 year survival rate of $86 \%{ }^{27}$ The Type II is aggressive tumor usually not associated with unopposed estrogen or hyperplasia, early invad-ing myometrium and lympho-vascular spaces with associated advanced-stage disease and higher tumor grade non-endometroid histology; and shows a high mortality rate with poorer prognosis. ${ }^{28}$ The GOG study group reported it's statistically result in large cohort of endo-metrial cancer patients, the clear cell carcinoma and papillary serous carcinoma has the worst prognosis, the 5 year disease free survival is $25 \%$ and $18 \%{ }^{24}$

There was a significant correlation between peritoneal cytology as prognostic factor in univariate analysis. In the past 20 years, over 50 reports on the significance of positive peri-toneal cytology in endometrial carcinoma have been published and many conflicting results have appeared in the literature several reports in the literature have noted increased recurrence rates and decreased survival rates and, on this basis, have recommended treatment for positive cytology. ${ }^{10,29-36}$ Positive peritoneal cytology was found to be associated with deep myometrial invasion, cervical involvement, adnexal spread, and lymph node metastasis as well as propensity for intra abdominal disease recurrence. Kadar et al, ${ }^{34}$ demonstrated that positive cytology did not influence survival if the disease was confined to the uterus using Cox's proportional hazards model.

Univariate and multivariate analysis showed there was a significant correlation between lymph node metastases as prognostic factors in this study. Lymph node spread represents the most common site of extrauterine disease in endometrial cancer. Reports show that 5 year survival for patients with stage I and II disease ranges from $80 \%$ to $91 \%$, and those patients with nodal metastases have a survival of only $44-52 \%$. $^{36,37}$

In univariate analysis there was a significant role between surgical procedure in prognosis but in multivariate Cox regression analysis it could be entered as it is decided by the stage, type and depth of myometrial invasion and lymphnode metastases. The choice of treatment method depends upon patient's willful demand and condition 
and current economic condition etc. Many studies have demonstrated that the survival rate does not vary between simple total hysterectomy and radical hysterectomy and thus the type of hysterectomy is not a dependent prognostic factor. ${ }^{9,38}$ It has been reported that radical hysterectomy should be considered when gross cervical involvement is encountered ${ }^{20,39}$ The subsequent change in the FIGO staging system in $1988^{40}$ recognizes that pelvic lymph node metastasis may be present in approximately $8 \%-10 \%$ of apparent stage I tumors and reflects the influence of depth of myometrial invasion on this risk. Omentectomy does not form part of the FIGO staging for endometrial cancer, yet omental biopsy or full omentectomy is frequently performed in cases of uterine serous carcinoma.

This study did not show a strong association between adjuvant therapy and improved survival. The evidence continues to support a role for adjuvant radiotherapy in the treatment of early endometrial cancer for women at high risk of loco-regional recurrence but not for intermediate risk and distant recurrences are not prevented by this strategy and there may be a future role for adjuvant chemotherapy. Currently there is insufficient evidence of a survival benefit to support the routine use of adjuvant therapy. Data from adequate randomized trials are needed to determine the benefit of chemo-radiation and chemotherapy either prior to or following radiation. In endometrial cancer, there is currently no proof that adjuvant hormone therapy or chemotherapy results in better outcome. Adjuvant radiotherapy, while effective at reducing rates of loco-regional recurrence, does not alter survival. ${ }^{41}$ Radiotherapy has been widely used as adjuvant treatment following surgery in women at increased risk of disease recurrence. The results from Post-Operative Radiotherapy in Endometrial Cancer (PORTEC 1), one of a number one completed randomized trials addressing the survival, showed that post-operative radiotherapy in stage 1 endometrial cancer reduced locoregional recurrence from $14 \%$ to $10 \%$ but had no impact on overall survival. ${ }^{42}$

\section{Conclusion}

In this study univariate model revealed menopausal status, the FIGO stage, grade, histologi-cal type, myometrial invasion, ER, PR, peritoneal cytology, lymph node metastases, adjuvant therapy, and the method of operation were related with the prognosis significantly. The multivariate analysis Cox proportion hazards regression model showed that the ER $(\mathrm{P}=0.004), \mathrm{PR}(\mathrm{P}=0.000)$, myometrial invasion $(\mathrm{P}=0.006)$ and lymph node metastases $(\mathrm{P}=0.049)$ were related with the prognosis significantly. The clinicopathological character of endometrial cancer is responsible for patient's better survival. If the patients had early detection, and treated in proper modalities, this might improve the prognosis.

Conflict of interest: The authors declare that they have no conflict of interests.

\section{References}

1. Parkin DM, Whelan SL, J. Ferlay J, et al. Cancer incidence in five continents. IARC Scientific. Publication. Lyon France: International Agency for Research on Cancer. 1997; 143:1-1240.

2. Pisani P, Parkin DM, Ferlay, J. Estimates of the worldwide mortality from eighteen major cancers in 1985. Implications for prevention and projections of future burden. Int J Can-cer, 1993; 55: 891-903.

3. Coleman MP, Esteve, J, Damiecki, P ,et al. Cancer Incidence and Mortality. IARC Sci.Publ. Lyon, France: International Agency for Research on Cancer. 1993: $1-806$.

4. G. Cannon, ed. Food, Nutrition and the Prevention of Cancer: A Global Perspective, Washington DC: World Cancer Research Fund. American Institute for Cancer Research 1997; 36-52.

5. Deligdisch L, Holinka CF. Endometrial carcinoma: Two diseases? Cancer Detect Preven-tion. 1987; 10: 237-246.

6. Bokhman JV. Two pathogenic types of endometrial carcinoma. Gynecol Oncol. 1983; 5: 0-17.

7. Boronow RC, Morrow CP, Creasman WT, et al. Surgical staging in endometrial cancer; Clinical pathological findings of a prospective study. Obst Gynecol. 1984; 63: 825-32.

8. Di Saia PJ, Creasman WT, boronow RC, et al. Risk factors and recurrent patterns in stage I endometrial cancer. Am J Obstet Gynecol. 1985; 151: 1009-15.

9. Creasman WT, Morrow CP, Bundy BN, et al. Surgical pathological spread patterns of endometrial cancer. A gynecologic Oncology Group study. Cancer, 1987; 60: 2035-41.

10. Morrow CP, Bundy BN, Kurman RJ, et al. Relationship between surgical pathological risk factors and outcome in clinical stage I and II carcinoma of the endometrium A gy-necologic Oncology Group study. Gynecol Oncol. 1991; 40: 55-65.

11. Mundt AJ, Waggoner S, Yamada D et al. Age as prognostic factor for recurrence in pa-tients with endometrial carcinoma. Gynecol Oncol, 2000; 79(1): 79-85. 
12. Farley JH, Nycum LR, Birrer MJ, Park RC, Taylor RR. Age-specific survival of women with endometriod adenocarcinoma of the uterus. Gynecol Oncol, 2000; 79: 86-9.

13. Salvesen HB, Akslen LA, Albrektsen G, et al. Poorer survival of nulliparous women with endometrial carcinoma. Cancer, 1998; 82: 1328-33.

14. Fuberg AS, Thune I. Metabolic abnormalities (hypertension, hyperglycemia and over-weight), lifestyle (high energy intake and physical inactivity) and endometrial cancer risk in a Norwegian cohort. Int J Cancer. 2003; 104: 669-76.

15. Calle EE, Kaaks R: Overweight, obesity and cancer: epidemiological evidence and pro-posed mechanisms. Nat Rev Cancer 2003; 4: 579-91.

16. Calle EE, Rodriguez C, Walker-Thurmond K, Thun MJ. Overweight, obesity, and mor-tality from cancer in a prospectively studied cohort of U.S. adults. N Engl J Med. 2003; 348: 1625-38.

17. Nakanishi T, Ishikawa H, Suzuki Y, et al. Association between menopausal state and prognosis of endometrial cancer. Int J Gynecol Cancer, 2001; 11: 483-87.

18. Anderson KE, Anderson E, Mink PJ, et al. Diabetes and endometrial cancer in the Iowa women's health study. Cancer Epidemiol Biomarkers Prev, 2001; 10: 611-17.

19. Steiner E, Eicher O, Hofmann M. Endometrial carcinoma in patients with diabetes melli-tus]. Zentralbl Gynakol, 2001; 123: 622-27.

20. Boente MP, Orandi YA, Yordan El et al. Recurrence patterns and complications in endo-metrial adenocarcinoma with cervical involvement. Ann Surg Oncol, 1995; 2: 138 -44.

21. Caroll K. FIGO stage, histology, histologic grade, age and race as prognostic factors in determining survival for cancer of the female gynecological system: an analysis of 1973-1987.

22. Zaino RJ, Kurman RJ, Herbold D et al. The significance of squamous differentiation in endometrial carcinoma. Cancer. 1991; 68: 2293-2302.

23. Abeler VM, Kjorstad KE. Endometrial adenocarcinoma in Norway. A study of a total population. Cancer. 1991; 67: 3093-3103.

24. Zaino RJ, Kurman RJ, Diana KL et al. Pathologic models to predict outcome for women with endometrial adenocarcinoma. Cancer. 1996; 77: 1115-21.
25. Jeon YT, Park IA, Kim YB et al. Steroid receptor expressions in endometrial cancer: Clinical significance and epidemiological implication. Cancer Lett. 2006; 239: 198-204.

26. Sivridis E, Giatromanolaki A, Koukourakis M, et al.. Endometrial carcinoma: Association of steroid hormone receptor expression with low angiogenesis and bcl-2 expression. Virchows Arch, 2001; 438: 470-77.

27. Gates EJ, Hirschfield L, Matthews RP, et al. Body mass index as a prognostic factor in endometrioid adenocarcinoma of the endometrium. J Natl Med Assoc. 2006; 98: 1814-22.

28. Bokhman JV. Two pathogenetic types of endometrial carcinoma. Gynecol Oncl. 1983; 15: 10-17.

29. Mazurka JL, Krepart GV, Lotocki RJ. Prognostic significance of positive peritoneal cytology in endometrial carcinoma. Am J Obstet Gynecol. 1988; 158: 303-6.

30. Turner DA, Gershenson DM, Atkinson N, et al. The prognostic significance of peritoneal cytology for stage I endometrial cancer[. Obstet Gynecol. 1989; 74: 775-80.

31. Obermair A, Geramou M, Tripcony L, et al. Peritoneal cytology: impact on disease-free survival in clinical stage I endometrioid adenocarcinoma of the uterus. Cancer Lett. 2001; 164: $105-110$.

32. McLellan R, Dillon MB, Currie JL, et al. Peritoneal cytology in endometrial cancer: a review. Obstet Gynecol Surv. 1989; 44: 711-19.

33. Lurain JR. The significance of positive peritoneal cytology in endometrial cancer Gynecol Oncol, 1992; 46: 143-44.

34. Kadar N, Homesley HD, Malfetano JH. Positive peritoneal cytology is an adverse factor in endometrial carcinoma only if there is other evidence of extrauterine disease. Gynecol Oncol, 1992; 46: 145-49.

35. Grimshaw RN, Tupper WC, Fraser RC, et al. Prognostic value of peritoneal cytology in endometrial carcinoma. Gynecol Oncol. 1990; 36: 97 - 100.

36. Creasman WT, Odicino F, Maisonneuve $\mathrm{P}$ et al. Carcinoma of the corpus uteri. Int J Gy-naecol Obstet, 2003; 83 (Suppl 1): 79-118.

37. Partridge EE, Shingleton HM, Menck HR. The National Cancer Data Base report on en-dometrial cancer. J Surg Oncol, 1996; 61: 111-23.

38. Hareyama H, Sakuragi N, Mishiya N, et al. Analysis 
of operative procedure and adjuvant therapy for endometrial carcinoma. J Jpn.Soc.Cancer Ther. 1997; 32: $115-23$.

39. Martin JD, Hahnel R, Mc Cartney Aj, et al. The effect of estrogen receptor status on sur-vival in patients with endometrial cancer. Am J Obstet Gynecol, 1983; 147: 322-24.

40. International Federation of Gynecology and obstetrics (FIGO): Announcements. FIGO stages: 1998 revision. Gynecol Oncol , 1999; 35: 125-27.

41. Keys HM, Roberts JA, Brunetto VL, et al. A phase III trial of surgery with or without adjunctive external pelvic radiation therapy in intermediate risk endometrial adenocarci-noma: A Gynecologic Oncology Group study. Gynecol Oncol, 2004; 92: 744-51.

42. Creutzberg CL, van Putten WLJ, Koper PCM, et al. Surgery and postoperative radiothe-rapy versus surgery alone for patients with stage-1 endometrial carcinoma: multicentre randomized trial. Lancet, 2000; 355: 1404-11. 